International Journal of Advanced Trends in Computer Science and Engineering

Available Online at http://www.warse.org/IJATCSE/static/pdf/file/ijatcse3091.12020.pdf

https://doi.org/10.30534/ijatcse/2020/3091.12020

\title{
Discrete Curvelet Transform Algorithm for Image Compression System
}

\author{
Noor Huda Ja'afar ${ }^{1}$, Syazmeer Sabudin ${ }^{2}$, Afandi Ahmad ${ }^{3}$ \\ ${ }^{1}$ Instrumentation and Control Engineering, Universiti Kuala Lumpur Malaysian Institute of Industrial \\ Technology, Malaysia \\ ${ }^{2}$ Instrumentation and Control Engineering, Universiti Kuala Lumpur Malaysian Institute of Industrial \\ Technology, Malaysia \\ ${ }^{3}$ Faculty of Electrical and Electronic Engineering, Universiti Tun Hussein Onn, Malaysia
}

\begin{abstract}
A medical image has dominated the compression techniques since it requires to have a smaller size of the image and should maintain the relevant diagnostic information. Reducing the size of the image, directly give impact to the transmission speed. This paper presents an approach towards compression algorithm development using discrete curvelet transform. Curvelet functions signify functions which have discontinuities along straight lines where wavelet functions are not properly worked. The curvelet transform algorithm were synthesized using MATLAB and various medical modalities such as computed tomography (CT), magnetic resonance imaging (MRI) and ultrasound are used as a sample's medical images. The results obtained clearly identify that discrete curvelet transform algorithm gives a good quality for the reconstructed images.
\end{abstract}

Keywords: Curvelet transform, MATLAB, medical image compression.

\section{INTRODUCTION}

In a high-tech world, various types of medical imaging modalities are used to assist diagnosis and analyze the diseases inside the human body [1]-[3]. The increasing number of patients annually will potentially increase the medical data generated. In addition, a fact of the standard image has a smaller size compared to the medical image has proved that a large data storage is required in medical imaging applications.

The higher usage of medical imaging contributes a massive amount of volumetric data for storage. The growing amount of medical image data is overwhelming over the world. In essence, assume if the size of the medical data is increasing every year. Therefore, to transmit a large size of medical image with limited speed range will requires more than one second. Moreover, a big data storage is needed to store the image data for further analyzing [4].
A novel image compression technique using features of curvelet transforms is proposed to advance the efficiency and compression performance of the medical imaging. Certainly, the curvelet transform is a non-adaptive technique developed for multiscale transforms. It was developed to overcome the limitations of the wavelet transform and become a well-liked algorithm especially in signal and image processing system. Curvelets was developed to address the edge representation problem where it is more efficient in representing edges and other singularities along the curve compared to wavelet.

\section{CURVELET VERSUS WAVELET TRANSFORM}

Due to limitation on wavelet, curvelet was developed to overcome the limitations on wavelet transform. The interesting characteristics about curvelet transform are:

i. Anisotropic scaling principle.

ii. Multiscale geometric transform.

iii. Special microlocal features.

Figure 1 depicts the main differences between curvelet and wavelet transform. As shown in Figure 1(a), there are a lot of coefficients required to covers for the edges. Whilst, less coefficients are needed to account the edges as depicted in Figure 1(b).

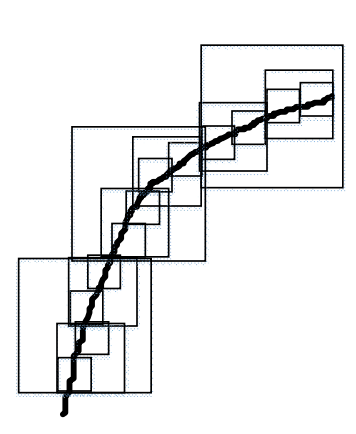

(a)

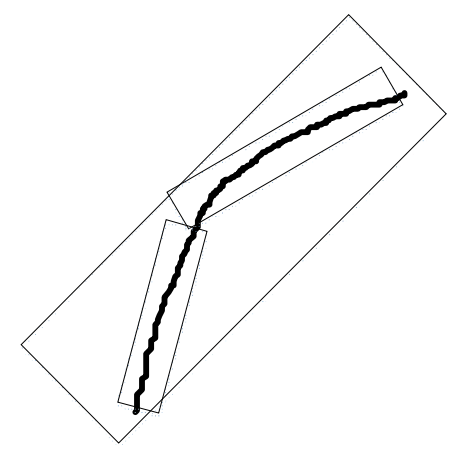

(b)
Figure 1: The different between wavelet and curvelet in sparse approximation 
Noor Huda Ja'afar et al., International Journal of Advanced Trends in Computer Science and Engineering, 9(1.1), 2020,166 - 169

\section{THEORY OF CURVELET TRANSFORM}

\subsection{First Generation}

The first generation of curvelet transform is called Continuous Curvelet Transform, where it used a complex algorithm of Ridgelet transform of an image [5]. The algorithm was modified in 2003 due to slow performance. Thus, to increase the speed and reduce the redundancy of the transform, the use of the Ridgelet transform is eliminated. In general, all curvelet transform may fall into one of these three categories [6]:

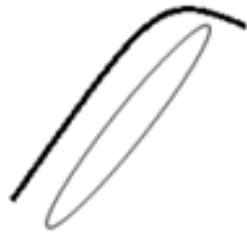

(a)

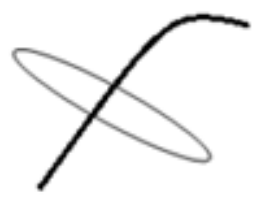

(b)

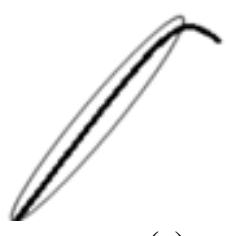

(c)
Figure 2: Category of curvelet transform

(a) Magnitude is equal to zero, when there is no intersect between the length-wise supports with the discontinuity.

(b) Magnitude is close to zero, when there is an intersect between the length-wise support with the discontinuity.

(c) Magnitude is larger than zero, when there is an intersect between the length-wise support with the tangent of the discontinuity.

\subsection{Second Generation}

The second generation curvelet transform is called as Fast Discrete Curvelet Transform (FDCT). There are two methods than can be used in FDCT implementations which are Unequally Spaced Fast Fourier Transform (USFFT) and frequency wrapping. Even though both methods will give the same output, but the frequency wrapping method offers a fast implementation.

Essentially, Fast Fourier transform (FFT) algorithm consist in the discrete curvelet transform process and it has a multi-resolution analysis. In addition, the convolution process in the discrete curvelet transform involves the Fourier domain. After the discrete curvelet transform computation process is done, the curvelet coefficients are generated. To get the curvelet coefficients, both forward and inverse FFT are utilized in the computations [7].

A series of translations is touch with the frequency tiles before the inverse Fourier domain is applied [8-11]. As illustrated in Figure 3, the spectral partitioning method is done to generate the curvelet coefficients. It contains three sections which are coarse, detail and fine. The low, intermediate and high frequencies allocated at the rough, detailed and soft levels respectively.

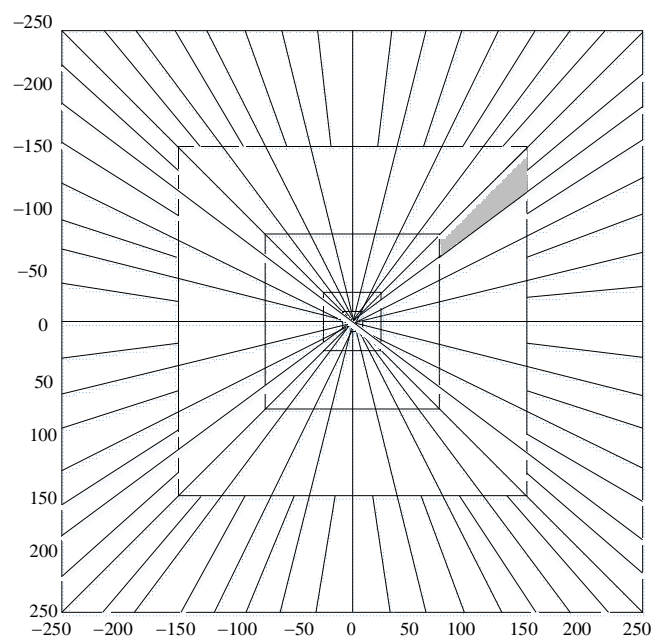

Figure 4: Curvelet based on frequency wrapping technique

\subsection{Wrapping-based Discrete Curvelet Transform}

Generally, the wrapping method use a decimated rectangular grid and has faster computation time compared to USFFT method. The oversampled curvelet coefficients is defined by:

$$
c^{D, O}(j, \ell, k)=\frac{1}{n^{2}} \sum_{n_{1}, n_{2} \in R_{j}, \ell} \hat{f}\left[n_{1}, n_{1}\right] \widetilde{U}_{j, \ell}\left[n_{1}, n_{2}\right] \mathrm{e}^{2 \pi i\left(k_{1} n_{1} / R_{1, j}+k_{2} n_{2} / R_{2, j}\right)}
$$

where the symbol $O$ and $D$ represents Oversampled and Digital respectively. A rectangle of size $R_{l, \ell} \times R_{2, \ell}$ and consists $P_{j, \ell}$ of are represented as $R_{j, \ell}$ symbol. The coefficients $c^{D, P}(j, \ell, k)$ is generated by assuming the $R_{1, \ell}$ and $R_{2, \ell}$ divide the image of size $n$. Then, the convolution process of curvelet and signal $f\left(t_{1}, t_{2}\right)$ are applied. Furthermore, the curvelet coefficients are down-sampled and relabeling the frequency samples of the form:

$$
\begin{aligned}
& n_{1}^{\prime}=n_{1}+m_{1} L_{1, j} \\
& n_{2}^{\prime}=n_{2}+m_{2} L_{2, j}
\end{aligned}
$$

Generally, the steps for the curvelet wrapping-based method is described as follows:

(a) 2D FFT is applied to the input image.

(b) Divide the interval of the frequency into digital corona tiles.

(c) For each corona tiles:

(i) Translate the tile by performing the multiplication of polar wedge with the Fourier samples.

(ii) Wrapping the parallelogram shaped which is formed by a tile around a rectangle at the origin.

(iii) Apply inverse FFT.

(iv) Sum the curvelet array to the collection of curvelet coefficients. 


\subsection{Image Compression Algorithm Based on Curvelet Transform}

Three basic processes of image compression include transformation, quantization and entropy coding. This paper focuses on the transformation part which explores the advantages of curvelet transform. Moreover, by using FDCT, the curvelet coefficients were generated from the original image decomposition. Figure 5 illustrates the proposed architecture for medical image compression system. The curvelet transform coefficients are generated using the wrapped method.

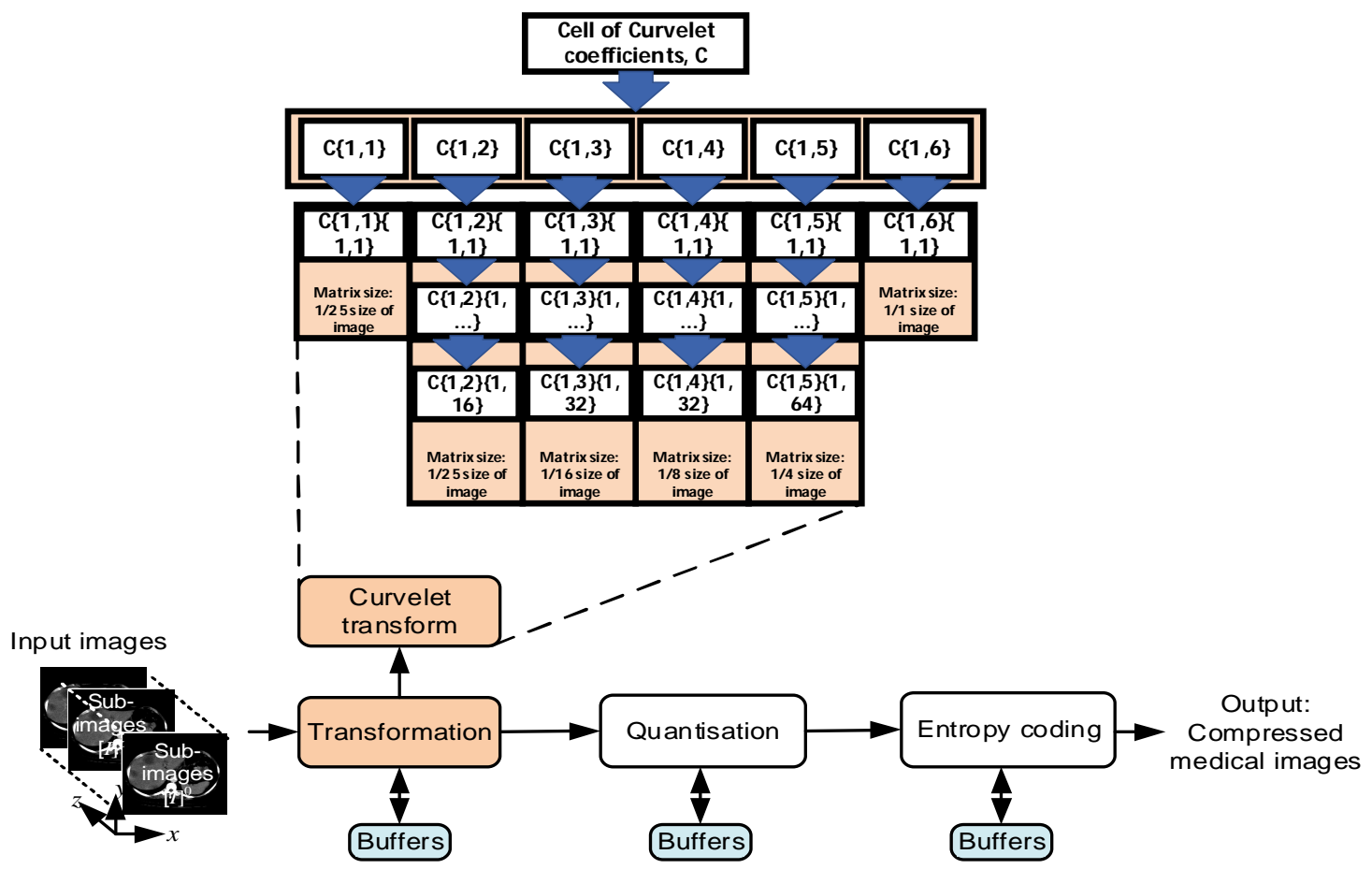

Figure 5: The proposed system using curvelet transform algorithm

\section{RESULTS AND DISCUSSION}

Two parameters are used to evaluate the simulation results which are MSE and PSNR. Moreover, the quality of the reconstructed image is compared with the original image. The value of MSE is calculated using the following formula:

$$
M S E=\frac{1}{M N}\left[y(i, j) \backslash y^{(i, j)}\right]^{2}
$$

Whilst, the PSNR of a gray scale image with size of 8-bits per pixel is defined as:

$$
P S N R=10 \log _{10}\left[\frac{255^{2}}{M S E}\right]
$$

Various type of medical images such as CT, MRI and ultrasound was used to synthesize the algorithm development in MATLAB ${ }^{\circledR}$. Figure 6 (a)-(c) shows the simulation results whilst Table I records the performance values in terms of MSE and PSNR.
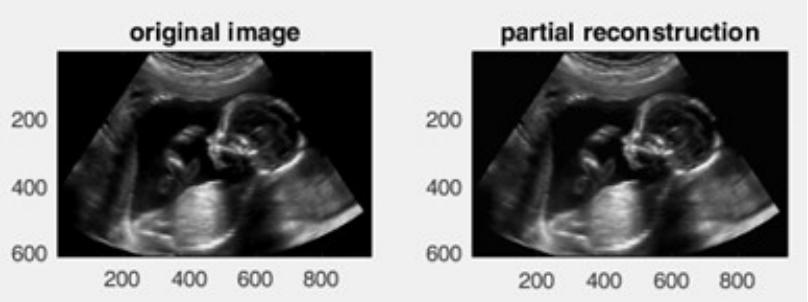

(a) Ultrasound fetus image
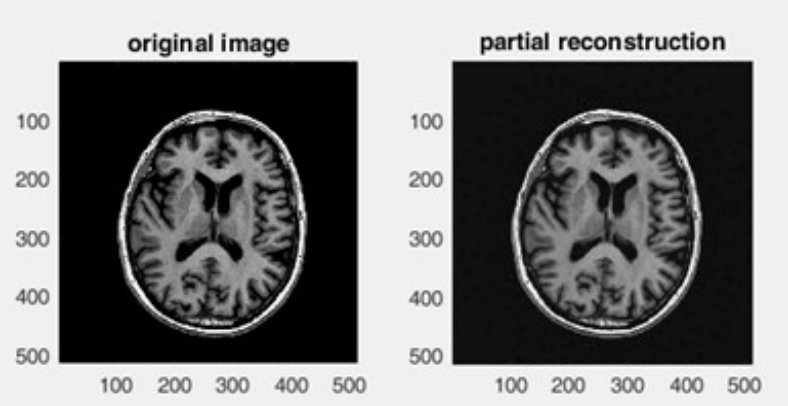

(b) MRI brain image 


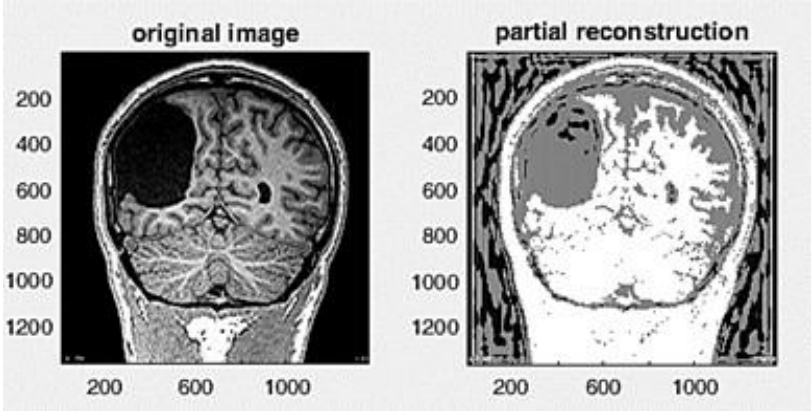

(c) CT brain image

Figure 6: Simulation results

Table I: Parameter Evaluations

\begin{tabular}{|c|c|c|}
\hline Images & MSE & PSNR (dB) \\
\hline Ultrasound fetus & 1.64 & 46.02 \\
\hline MRI brain & 28.86 & 33.56 \\
\hline CT brain & 36.44 & 19.49 \\
\hline
\end{tabular}

The results shown in Table I clearly indicates that curvelet transform algorithm provides higher PSNR values. Moreover, the quality of the reconstructed images is acceptable as illustrated in Figure 6.

\section{CONCLUSION}

In this paper, a wrapping-based of discrete curvelet transform method was proposed for medical image compression system. Although this paper presents a review of curvelet transform comprehensive insight into the different applications where it is being applied to. With more passionate research and expansion of scope, curvelets can be applied to diverse fields especially on medical imaging, so that revolutionary results can be obtained. Various types of medical images are used and verify that the discrete curvelet transform algorithms are suitable to be implemented with medical images.

\section{REFERENCES}

1. S. Safie, et al, "Bipolar pulse active features of ECG biometric application," International Conference on Biosignal Analysis, Processing and System (ICBAPS), Kuala Lumpur, 2015, pp. 1-5. https://doi.org/10.1109/ICBAPS.2015.7292207

2. S. Safie, et al, "Comparison of pulse active (PA) modulation signal for electrocardiogram (ECG) authentication," IEEE International Conference on Signal and Image Processing (ICSIPA), Kuala Lumpur, 2015, pp. 165-168. https://doi.org/10.1109/ICSIPA.2015.7412183

3. S. S. Mazlan, et al, "Development of anterior cruciate ligament (ACL) knee injury classification system from magnetic resonance image (MRI) using fuzzy inference system," Science and Engineering Technology National Conference (SETNC), Putrajaya, 2015.
4. Reddy, K. S. N., Reddy, B. R. S., Rajasekhar, G., \& Rao, K. C. (2012). A Fast Curvelet Transform Image Compression Algorithm using with Modified SPIHT. International Journal of Computer Science and Telecommunications, 3(2), 1-9.

5. Kourav, A., \& Singh, P. (2013). Review on Curvelet Transform and Its Applications. Asian Journal of Electrical Sciences, 2(1), 9-13.

6. Parmar, C. K., \& Pancholi, P. K. (n.d.). (2012). Image Compression Based on Curvelet Transform. 1(10), $1-5$.

7. Reddy, K. S. N., Reddy, B. R. S., Rajasekhar, G., \& Rao, K. C. (2012). A Fast Curvelet Transform Image Compression Algorithm using with Modified SPIHT. International Journal of Computer Science and Telecommunications, 3(2), 1-9.

8. S. P. Bingulac, "On the compatibility of adaptive controllers (Published Conference Proceedings style)," Proc. 4th Annu. Allerton Conf. Circuits and Systems Theory, New York, 1994, pp. 8-16.

9. Lawicki, T., \& Zhirnova, O. (2015). Application of curvelet transform for denoising of CT images. Photonics Applications in Astronomy, Communications, Industry, and High-Energy Physics Experiments 2015, 9662, 966226.

https://doi.org/10.1117/12.2205483

10. Anandan, P., \& Sabeenian, R. S. (2016). Medical Image Compression Using Wrapping Based Fast Discrete Curvelet Transform and Arithmetic Coding. Circuits and Systems, 7(8), 2059-2069.

https://doi.org/10.4236/cs.2016.78179

11. Preda, R. O., \& Vizireanu, N. D. (2011). Quantisation-based video watermarking in the wavelet domain with spatial and temporal redundancy. International Journal of Electronics, 98(3), 393-405.

https://doi.org/10.1080/00207217.2010.547810 